\title{
Personalidad, edad y rendimiento deportivo en jugadores de fútbol desde el modelo de Costa y McCrae
}

\author{
Roberto Ruiz-Barquín ${ }^{1}$ y Alejo García-Naveira ${ }^{2}$ \\ 1 Universidad Autónoma de Madrid \\ 2 Centro de Estudios e Investigación (CEI), Club Atlético de Madrid
}

\begin{abstract}
Resumen: El estudio de la personalidad en el deporte de rendimiento sigue constituyéndose como una importante línea de investigación. En la última década, se han obtenido algunos resultados confirmatorios, aunque no concluyentes, respecto al estudio de relación entre la personalidad, la edad y el rendimiento de los deportistas, recomendándose más estudios al respecto para esclarecer este panorama. Con esta intención, los objetivos que se persigue en el presente estudio son: 1.- Establecer posibles diferencias en personalidad en función de la categoría por edad deportiva y 2.- Estudiar la relación entre la personalidad y el rendimiento deportivo, y establecer la capacidad predictiva del Modelo de los Cinco Factores de Personalidad respecto al rendimiento. Para ello, se ha aplicado el cuestionario de personalidad NEO-FFI (Costa y McCrae, 2008) y evaluado el rendimiento deportivo a una muestra de 128 jugadores de fútbol con edades comprendidas entre los 14 y 24 años. Los resultados indican que los deportistas pertenecientes a la categoría de adultos obtienen mayores puntuaciones en los rasgos Apertura a la Experiencia y Responsabilidad que los de las categorías juveniles y cadetes, mientras que los juveniles tienen un mayor rasgo de Neuroticismo que los adultos. Además, para la muestra total de deportistas, los datos indican que los rasgos Apertura a la Experiencia y Responsabilidad se relacionan positivamente con el rendimiento deportivo, mientras que el rasgo Neuroticismo lo hace de forma negativa. En este caso se obtiene modelos de regresión significativos $(p<.05)$ con una capacidad predictiva del 5.1\% introduciendo las variables Neuroticismo y Apertura a la Experiencia. Respecto a la categoría por edad, sólo se muestran modelos de regresión con tendencia a la significación estadística $(p<.10)$ en la categoría cadete. Sin embargo, incluyendo en los modelos de regresión la variable edad del futbolista, se obtienen modelos de regresión lineal significativos de forma selectiva para los futbolistas de 14 años y mayores de 18 años $(p<.05)$, llegando a predicciones del $24.3 \%$ y $25.6 \%$ respectivamente. Las variables introducidas en el modelo de regresión para los futbolistas de 14 años son Neuroticismo y Reponsabilidad, y Neuroticismo y Extraversión para los futbolistas mayores de 18 años. Estos datos apoyan tanto a la "hipótesis de selección" e "hipótesis de desarrollo" como la "perspectiva crédula" del estudio de la personalidad en el contexto deportivo.

Palabras claves: Personalidad; categoría por edad; alto rendimiento; hipó-
\end{abstract}

Title: Personality, age and athletic performance in soccer players from the model of Costa and McCrae.

Abstract: The study of personality in performance is still an important line of research. In the last decade, some confirmatory results have been obtained, though they are not conclusive, with regard to the study of relation between personality, age and performance of athletes. Further conclusive studies are needed. With this intention, the aims pursued in this study are: 1.-To establish possible differences in personality depending on the category of sports age and 2.- To study the relation between personality and sports performance, and establish the capacity Model predictive Five Personality Factors regarding performance. In order to accomplish this, the questionnaire of personality NEO-FFI (Costa and McCrae, 2008) has been applied and the sports performance has been evaluated with a sample of 128 football players aged between 14 and 24. The results indicate that the athletes belonging to the adults' category obtain higher scores in the traits Opening to the Experience and Responsibility than those of the youth and junior categories, while juveniles have a higher trait of neuroticism than adults. In addition, the traits Opening to the Experience and Responsibility relate positively to the sports performance, whereas the trait Neuroticism does it in a negative way. In this case, results in significant regression models $(p<.05)$ with a $5.1 \%$ predictive variables entering Neuroticism and Openness to Experience. Regarding age category, only regression models with trend to statistical significance ( $p$ $<.10)$ are shown in the cadet category. However, by including the variable age of football players in the regression models, we get significant linear regression models selectively for football players aged 14 and over 18 ( $p$ $<.05$ ), achieving predictions of $24.3 \%$ and $25.6 \%$ respectively. The input variables in the regression model for football players aged 14 are Neuroticism and Responsibility, and Neuroticism and Extraversion for football players over 18. This information supports the "hypothesis of selection" and "hypothesis of development" and the "credulous perspective" of the study of the personality on the sports context.

Key words: Personality; age category; high performance; selection hypothesis; hypothesis development; credulous perspective.

tesis de selección; hipótesis de desarrollo; perspectiva crédula.

\section{Introducción}

El estudio de la personalidad en el deporte sigue constituyéndose como una importante línea de investigación (Cox, 2009; Vealey, 2002; Weinberg y Gould, 2010), en la que los trabajos están orientados principalmente a responder a dos categorías de preguntas (Valdés, 1998). En la primera categoría, las preguntas se refieren a la influencia del deporte (de la participación en) sobre la personalidad. Por ejemplo, ¿se produce un cambio en los rasgos de personalidad del individuo como consecuencia de su participación en el deporte? En la segunda categoría, las preguntas se corresponden a la tentativa de predecir diferencias individuales en la participa-

* Dirección para correspondencia [Correspondence address]: Roberto Ruiz-Barquín. Universidad Autónoma de Madrid. Facultad de Formación de Profesorado y Educación. Calle Francisco Tomás y Valiente, 3. Campus de Cantoblanco. 28049. Madrid. (España).

E-mail: roberto.ruiz@,uam.es ción en el deporte y en el rendimiento deportivo y, finalmente, de explicarlas en términos de la personalidad o de los rasgos de personalidad. Por ejemplo, ¿existen diferencias en personalidad en función de la práctica o no deportiva, modalidad deportiva (individual vs. equipo), del género o rendimiento de los deportistas?

Realizando una reflexión de los estudios revisados, esta área se encuentra en un período de progreso y los diferentes trabajos van subsanando las limitaciones metodológicas que a lo largo de la historia han sido motivo de crítica, como son la variedad de teorías e instrumentos utilizados en el análisis de la personalidad, la dificultad de constituir la muestra de estudio, la falta de replicabilidad de las investigaciones y la falta de estudios inter-culturales (García-Naveira, 2010; Ruiz, 2004; Valdés, 1998). Por ejemplo, en la actualidad el modelo de los Cinco Grandes factores de personalidad se ha impuesto y existe un número importante de instrumentos diseñados para su medida, de los cuales el más prototípico es el NEO- 
PI-R, Revised Neo Personality Inventory (Costa y McCrae, 1992), en el que se recomienda su uso en todos aquellos contextos evaluativos en los que es conveniente medir la personalidad (p.ej., clínico, educativo, organizacional o deportivo), pues en los últimos veinte años este modelo se ha aceptado como la clasificación de rasgos de personalidad más validada y consensuada (Colegio Oficial de Psicólogos de España, 2011).

Desde esta perspectiva, cada vez son más los trabajos que ofrecen resultados clarificadores, aunque no concluyentes, por lo que se sugiere continuar investigando al respecto. Por ejemplo, una de las conclusiones a las que llegan Rhodes y Smith (2006) y Hoyt, Rhodes, Hausenblas y Giacobbi (2009) es que la práctica deportiva se asocia positivamente con los rasgos de Responsabilidad (p.ej., tendencia al orden, autodisciplina y orientación al logro) y Extraversión (p. ej., sociabilidad, actividad, búsqueda de estimulación y afecto positivo) y negativamente con el rasgo Neuroticismo (p.ej., inestabilidad emocional, ansiedad y vulnerabilidad a la depresión).

También, en la última década, se han obtenido algunos resultados confirmatorios, tanto en el análisis de la evolución de la personalidad en función de la edad de los deportistas como en el estudio de la personalidad y el rendimiento deportivo, recomendándose más estudios en relación a esta temática para aclarar este panorama (García-Naveira, Ruiz y Pujals, 2011; Ruiz, 2005; Ruiz, 2012). Estas sub-áreas de estudio (edad y rendimiento) cobran un especial interés debido a su trascendencia aplicada (predicción del rendimiento, detección de talentos, programas de intervención, etc.). A continuación se revisan algunos trabajos representativos sobre la temática, y posteriormente, se investigan los aspectos anteriormente citados con el objetivo de contribuir a profundizar en el estudio de la personalidad en el contexto deportivo.

\section{Personalidad y diferencias por edad}

Un aspecto a tener en cuenta es la consideración de la participación en la práctica deportiva como uno de los factores que influye en las diferencias en personalidad o si las diferencias preexisten y las personas seleccionan su deporte de acuerdo a sus características de personalidad (Bakker, Whiting y Van Der Drug, 1993; Marín et al., 2012; Wann, 1997). Cuando las características en personalidad de los deportistas hacen que seleccionen determinados deportes se denomina hipótesis de selección (por ejemplo, un deportista extravertido decide participar en un deporte de equipo ya que promueve las relaciones entre sus integrantes), en la que los perfiles de personalidad de los participantes de cada deporte son generalmente compartidos. Por el contrario, el planteamiento teórico que defiende que la actividad deportiva influye sobre la personalidad del deportista se denomina hipótesis del desarrollo (por ejemplo, un deportista introvertido puede ser menos introvertido debido a la influencia de participar en un deporte de equipo). A continuación se realiza una revisión de trabajos representativos sobre ambas posturas.
Desde la "hipótesis de selección", diferentes resultados indican que los deportes atraen a cierto perfil de personas (Elman y McKelvie, 2003). Así, Gabler (1976) midió la evolución de la personalidad en el deporte. Mediante el $16 \mathrm{PF}$ estudió durante 5 años la personalidad de 154 nadadores adultos de élite (hombres y mujeres). Este autor concluye que la personalidad no varía a través del tiempo. Más recientemente, McKelvie, Lemieux y Scout (2003) utilizaron el EPQ de Eysenck en una muestra de 86 deportistas universitarios y no observaron variación en la personalidad tras un seguimiento de 4 años.

Respecto a la "hipótesis de desarrollo", uno de los primeros trabajos sobre la evolución de la personalidad de los deportistas lo encontramos en el estudio de Tattersfield (1971). Este autor ha proporcionado pruebas longitudinales de que la participación en los deportes antes de alcanzar la madurez influye en el desarrollo de la personalidad. Específicamente, evaluó los perfiles de personalidad de un grupo de jóvenes que participaban en un programa de natación durante un período de entrenamiento de cinco años. Durante este período se observó que los jóvenes presentaban cambios significativos hacia una mayor extraversión, estabilidad emocional y dependencia.

Más recientemente, Rodríguez (2003) llevó a cabo un estudio transversal de la personalidad con deportistas ( $\mathrm{n}=$ 1196) de distintos deportes (fútbol, artes marciales, atletismo, baloncesto y natación), sexo (hombres y mujeres) y edades (niños, jóvenes y adultos), a los que aplicó la Batería de Test Psicológicos para Deportistas de Competición (Fernández, Fernández y Mielgo, 1999). Los resultados indican que la edad de los deportistas juega un papel destacado en el desarrollo de la personalidad, de manera que en la medida que aumenta la edad también lo hacen los componentes psicológicos de la personalidad deportiva (autocontrol, control de la ansiedad, autoestima, autoeficacia, cohesión y cooperación).

Ruiz (2005) utilizó el Big Five Questionnaire (BFQ) en su versión en español (Bermúdez, 1995) a una muestra de 346 judokas españoles (234 de categoría masculina y 112 de categoría femenina) de competición nacional. La muestra estaba repartida en 4 categorías según la edad: 21 hombres y 14 mujeres infantiles (13-14 años), 87 hombres y 45 mujeres cadetes (15-16 años), 64 hombres y 40 mujeres juniors (17-18 años) y 62 hombres y 13 mujeres seniors (mayores de 18 años). En cuanto a las diferencias según las categorías, en el género masculino, los seniors tienen mayores puntuaciones que los cadetes en las dimensiones Afabilidad y Tesón. Además, los seniors obtienen mayores puntuaciones que los infantiles, cadetes y juniors en la dimensión Estabilidad Emocional. Por último, se observa una tendencia a la significación $(p<.10)$ donde los seniors obtienen mayores puntuaciones que los cadetes en la dimensión Apertura Mental.

Ruiz (2005) concluye que la actividad deportiva es un factor importante en la evolución y desarrollo de la personalidad del deportista. Por tanto, el paso del joven deportista por experiencias y exigencias deportivas, puede incrementar 
los niveles de determinadas características de personalidad, si bien los factores evolutivos y madurativos pueden estar interviniendo en mayor o menor medida.

García-Naveira et al. (2011) aplicaron el Five Factor Inventory (NEO-FFI; Costa y McCrae, 2008) a 155 jugadores de fútbol de alto rendimiento con una edad comprendida entre los 14 y 24 años $(M=17.18, D . T=2.44)$ que entrenaban 4 o 5 días a la semana en sesiones de 2 horas. Los sujetos se repartían en 3 categorías según la edad: 44 cadetes (15-16 años), 67 juveniles (17-18 años) y 46 adultos (mayores de 18 años). Los jugadores cadetes competían en la categoría "Autonómica", los jugadores juveniles en las categorías "Liga Nacional" y "División de Honor" y los jugadores adultos en las categorías "Tercera División" y "Segunda División b". En cuanto a las diferencias en función de la categoría por edad, los resultados del ANOVA muestra como existen diferencias en los rasgos Neuroticismo $(p<.05)$, Apertura a la Experiencia $(p<.001)$ y Responsabilidad $(p<.05)$. En concreto, en cuanto al rasgo Neuroticismo, los jugadores adultos obtienen menores puntuaciones que los jugadores juveniles $(p<.05)$. Respecto al rasgo Apertura de la Experiencia, los jugadores adultos obtienen mayores puntuaciones que los jugadores juveniles $(p<.001)$ y cadetes $(p<.001)$. Analizando el rasgo Responsabilidad, los jugadores adultos obtienen mayores puntuaciones que los jugadores cadetes $(p<.05)$. Por último, no se obtienen diferencias en los rasgos Extraversión y Amabilidad en los grupos anteriormente citados.

Además de estas diferencias, destacar la jerarquización del tamaño del efecto de estos rasgos (según los criterios de Cohen, 1988) en los que la Apertura a la Experiencia estaría a un nivel superior con un efecto grande $(d=0.98$ para cadetes y $d=0.80$ para juveniles), frente al Neuroticismo $(d=$ 0.56 para juveniles) y la Responsabilidad ( $d=0.47$ para cadetes) con un efecto mediano.

García-Naveira et al. (2011) concluyen que lo más probable, a la vista del desarrollo teórico e investigador en personalidad, es que tanto la "selección" como el "desarrollo" en la práctica de un deporte influyan conjuntamente en el perfil de personalidad del individuo. Partiendo de esta premisa, principalmente, el desarrollo de la personalidad dentro del contexto deportivo toma sentido cuando se centra en el estudio de niños y jóvenes (p.ej., debido a las experiencias y aprendizajes deportivos que tienen a lo largo de las temporadas) y la estabilidad de la personalidad y la selección del deporte cuando se estudia a deportistas adultos.

En resumen, las dos hipótesis establecidas (selección y desarrollo) tienen argumentos a favor y en contra. En la revisión realizada, se han encontrado pocos trabajos al respecto, aunque los resultados sugieren que a medida que aumenta la edad en los más jóvenes, los deportistas son más extrovertidos, estables emocionalmente, responsables, amables y abiertos ante nuevas experiencias, mientras que la personalidad de los deportistas adultos se mantiene estable a lo largo del tiempo.

\section{Personalidad y rendimiento deportivo}

Williams y James (2001) señalan que el rendimiento y resultado deportivo de un deportista es multifactorial. El éxito deportivo dependerá de un conjunto de cualidades propias (habilidades, capacidades físicas, variables psicológicas, etc.) y ajenas del mismo (árbitro, terreno de juego, rival, etc.). Centrándose en las variables del individuo, los deportistas que han obtenido éxito o alto niveles de desempeño poseen destreza y talento para realizar bien una actividad específica y puede que se trate de personas con características de personalidad similares (Casis y Zumalabe, 2008). Por tanto, la Psicología del Deporte tiene como uno de sus objetivos de estudio aclarar si existen determinadas características de personalidad que son necesarias o convenientes para un nivel específico o asociado a un mayor rendimiento deportivo.

Desde una perspectiva crédula de estudio, los autores afirman que existe relación entre la personalidad y el rendimiento deportivo, donde los rasgos pueden ayudar a predecir la conducta y el éxito deportivo (Arbinaga y Caracuel, 2008; Marrero, Martin-Albo y Nuñez, 2000) aunque esta relación no es de causa y efecto e intervienen otros factores como el motivacional (Weinberg y Gould, 2010).

Principalmente, los investigadores han seguido dos líneas de trabajo al estudiar la relación entre personalidad y rendimiento deportivo (García-Naveira, 2010): a) el estudio de la personalidad de deportistas de élite o alto rendimiento y b) el estudio correlacional y/o predictivo del rendimiento del deportista (evaluado de forma objetiva mediante el registro de acciones y resultados deportivos o de forma subjetiva a través de la valoración del entrenador y/o deportista).

En cuanto a la primera área de investigación, GarcíaNaveira et al. (2011) realizaron una revisión de trabajos sobre personalidad y alto rendimiento. Los resultados indican que los deportistas de alto nivel son más extravertidos (Bushan y Agarwal, 1978; Daino, 1985; Egloff y Gruhn, 1996; Foster, 1977; Gabler, 1976; Kane, 1964; Kirkcaldy, 1982; Novotny y Petrak, 1983; Olgilvie 1968; Reyes, Raimundi y Gómez, 2012; Ruiz, 2004; Tutko y Olgilvie, 1969), responsables (Kane, 1964; Olgilvie, 1968; Ruiz, 2001, 2004; Tutko y Olgilvie, 1969) y estables emocionalmente (Daino, 1985; Gabler 1976; Kane, 1964; Kirkcaldy, 1982; Olgilvie, 1968; Ruiz, 2001, 2004; Tutko y Olgilvie 1969) que los deportistas de menor nivel o baremo de la población general del instrumento de evaluación utilizado.

Además, García-Naveira et al. (2011) analizaron las diferencias en personalidad en función del nivel competitivo de los deportistas (alto rendimiento vs, amateurs). El instrumento utilizado para estudiar la personalidad fue el NEO-FFI (Costa y McCrae, 2008). La muestra de jugadores de fútbol de alto rendimiento estaba constituida por 46 adultos con una edad comprendía entre los 19-24 años $(M=22.3, D . T$. $=$ 2.4) que entrenaban 4 o 5 días a la semana en sesiones de 2 horas y que competían en las categorías de "Tercera División" y "Segunda División b". La muestra de jugadores de fútbol amateurs estaba formada por 32 adultos con una edad 
comprendía entre los 19-28 años $(M=23.7, D . T .=2.7)$ que entrenaban 2 días a la semana en sesiones de 2 horas y que competían en la categoría "Segunda Regional". Los resultados indican que los jugadores de fútbol de alto rendimiento obtienen mayores puntuaciones en los rasgos Extraversión $(p<.01)$ y Responsabilidad $(p<.001)$ que los jugadores amateurs, mientras que estos obtienen mayores puntuaciones en el rasgo Neuroticismo $(p<.01)$. No se obtienen diferencias en los rasgos Apertura a la Experiencia y Amabilidad en los grupos anteriormente citados.

También, destacar la jerarquización del tamaño del efecto de estos rasgos (según los criterios de Cohen, 1988) en los que la Responsabilidad $(d=0.88)$ estaría a un nivel superior con un efecto grande, frente a la Extraversión $(d=0.65)$ y Neuroticismo $(d=0.54)$ con un efecto mediano. Estos datos podrían sugerir que determinadas características de personalidad son características de los deportistas de alto rendimiento.

Por su parte, Ruiz (2012) estudió los rasgos de personalidad de 96 deportistas que practican deportes de combate en la Universidad Autónoma de Madrid (66 hombres y 30 mujeres) mediante la aplicación del BFQ (Bermúdez, 1995). Los resultados indican que los deportistas obtienen mayores puntuaciones en las dimensiones de Estabilidad Emocional $(p<.001)$ y Apertura Mental $(p<.01)$ que el baremo de la población del instrumento de medida, teniendo en cuenta la edad y el género. Además, las mujeres obtuvieron mayores puntuaciones en la dimensión Energía $(p<.05)$ que la población de referencia.

Respecto a la relación entre la personalidad y las medidas de rendimiento, Morgan (1980, citado en Vanden Auweele, Cuyper, Van Mele y Rzewnicki, 1993) destaca como la personalidad, considerada como predictor del rendimiento, puede llegar a explicar entre un 20\% y $45 \%$ de varianza. Estos datos sugieren que la personalidad está relacionada con el propio rendimiento del deportista. A continuación, se realiza una revisión de algunos trabajos representativos sobre personalidad y rendimiento deportivo.

Graydon y Murphy (1995) utilizaron el Eysenck Personality Questionnaire (EPQ; Eysenck y Eysenck, 1975) y analizaron el rendimiento deportivo (resultado de la competición) en una muestra de 20 jugadores de tenis de mesa. Los deportistas extravertidos obtienen un mayor rendimiento que los introvertidos ante situaciones de presencia de público y viceversa ante la situación sin público. Estos autores concluyen que la dimensión de personalidad extraversión/introversión es un factor significativo en las investigaciones de facilitación social.

Piedmont, Hill y Blanco (1999) aplicaron el Revised Neo Personality Inventory (NEO-PI-R; Costa y McCrae, 1992) y evaluaron el rendimiento deportivo en una muestra de 79 jugadoras de fútbol femenino, con una edad comprendida entre 18 a 21 años. El rendimiento fue evaluado durante 8 partidos a través de unas dimensiones valoradas por el entrenador (capacidad de ser entrenado, habilidad deportiva, rendimiento del partido, jugar en equipo y rendimiento total) y mediante el análisis estadístico de determinadas acciones de juego (goles, asistencias, partidos jugados y tiros). Dentro del análisis correlacional, la dimensión Neuroticismo se asocia negativamente con la valoración total del rendimiento (tanto la valoración del entrenador como el registro de acciones deportivas), mientras que la dimensión Responsabilidad se relaciona de forma positiva. Además, el análisis de regresión indica que las dimensiones de personalidad como Neuroticismo y Responsabilidad explican el 23\% de la varianza del rendimiento valorado por los entrenadores, mientras que únicamente la dimensión Responsabilidad explica el $8 \%$ de la varianza del registro de las acciones deportivas. Por último, estos autores señalan que alguno de estos rasgos pueden tener tanto una relación directa con el rendimiento deportivo (sobre las propias tareas a desarrollar: presionar, realizar un pase bajo presión, etc.) como indirecta (aspectos que están presentes en torno a la práctica y contexto deportivo: las relaciones sociales, hábitos, etc.).

Karp (2000) aplicó el 16 Personality Factors (16 PF; Cattell, Cattell y Cattell, 1993) a una muestra de 126 jugadores de hockey y concluye que varios factores de personalidad son indicadores importantes del logro deportivo (a través de la valoración de las expectativas de los deportistas): a) altas puntuaciones en las escalas Razonamiento (escala B: pensamiento abstracto), Estabilidad (escala C: emocionalmente estable, adaptada y madura), Atrevimiento (escala H: emprendedora, atrevida y segura en lo social), bajas puntuaciones en las escalas Abstracción (escala M: práctica, con los pies en la tierra, realista), Privacidad (escala N: abierta, genuina, llana, natural), Apertura-Cambio (escala Q1: tradicional, apegada a la familia) y Tensión (escala Q4: relajada, plácida, paciente).

Ruiz (2006) utilizó el BFQ (Bermúdez, 1995) en una muestra de 34 judokas de competición de ambos sexos (19 hombres y 15 mujeres) con edades comprendidas entre los 13 y 16 años y analizó su relación con los resultados deportivos que obtenían durante una temporada. Los resultados indican que la dimensión Tesón (responsabilidad) está asociada positivamente con una mejor clasificación en los Campeonatos Nacionales para ambos sexos, mientras que la dimensión Energía (extraversión) también se relaciona positivamente, pero sólo en las mujeres. Estos datos indican que hay que tener presente la variable sexo al predecir el rendimiento deportivo de los deportistas. Además, en las mujeres, la dimensión Tesón es un predictor del resultado deportivo en un $31,1 \%$ de varianza, mientras que conjuntamente las dimensiones de Estabilidad Emocional, Tesón y Afabilidad explican el $47.9 \%$ de varianza.

Ruiz (2008) analizó la relación entre sub-dimensiones del BFQ y los resultados deportivos de una muestra de judokas (ambos sexos) pertenecientes a la categoría cadete. Los resultados indicaron que en la dimensión Tesón, destacan la subdimensiones Perseverancia y Escrupulosidad en mujeres y Escrupulosidad en hombres. En la dimensión Energía, se observa una tendencia hacia la significación en las subdimensiones Dinamismo y Dominancia en mujeres y Dinamismo en hombres. En la dimensión Afabilidad, se obtiene 
una tendencia hacia la significación en la subdimensión Cordialidad/Amabilidad en mujeres. En la dimensión Estabilidad Emocional, destaca una tenencia hacia la significación en la subdimensión Control de Emociones en hombres. Además, la subdimensión Perseverancia es un predictor del resultado deportivo en un $28.1 \%$ de varianza en mujeres y un $22.6 \%$ de varianza en hombres. Este autor concluye que las subdimensiones de los rasgos de personalidad se relacionan con la predicción del rendimiento deportivo, recomendándose su estudio debido a que aportan información diferencial y complementaria.

En resumen, tanto los trabajos que analizan la personalidad de los deportistas de alto rendimiento o élite como los que estudian la relación entre personalidad y medidas de rendimiento, tienden a indicar que la extraversión, responsabilidad y estabilidad emocional se asocian con un mayor desempeño deportivo. Una posible explicación de estos resultados es a través de la "pirámide deportiva" representada por Silva (1984, citado por Cox, 2009). Los deportistas de alto nivel (cúspide de la pirámide) tienen un perfil homogéneo de personalidad debido a que determinados rasgos son necesarios para alcanzar un alto rendimiento.

En conclusión, a pesar del volumen de trabajos realizados, este campo de estudio, demuestra ser aún muy amplio e insuficientemente explorado por la Psicología del Deporte y la Actividad Física (Bara Filho, Scipiao y Guillen, 2004). En general, se observa la necesidad de realizar trabajos que profundicen en estas cuestiones, en la que una de las características de la ciencia es investigar, plantear dudas e incluso cuando se dispone de datos consistentes. El presente trabajo pretende dar respuesta a una de las principales cuestiones que ha sido motivo de debate entre los investigadores en los últimos años, como es el estudio de la personalidad, la edad y el rendimiento de los deportistas.

Para ello, se tendrá en cuenta algunos aspectos metodológicos a subsanar que han sido motivo de crítica a lo largo de los años en el estudio de la personalidad en el deporte. Se pretende centralizar la investigación en el modelo de personalidad de Costa y McCrae (2008) como uno de los principales representantes de la teoría de rasgo, utilizar un adecuado número de participantes españoles en el estudio y realizar una evaluación longitudinal del rendimiento de los deportistas.

\section{Predicciones e hipótesis:}

Las hipótesis del trabajo de investigación desarrollado son las siguientes:

a) Diferencias en función de la categoría de edad deportiva:

1) Los deportistas adultos obtienen mayores puntuaciones en los rasgos de Responsabilidad, Amabilidad y Apertura a la Experiencia que los deportistas de menor categoría (cadetes y juveniles), mientras que estos tienen mayores puntuaciones en Neuroticismo que los adultos. b) Personalidad y rendimiento deportivo:

2) Los rasgos de Responsabilidad y Extraversión se asocian positivamente con el rendimiento deportivo, mientras que el Neuroticismo lo hace de forma negativa.

\section{Método}

\section{Participantes}

La muestra está constituida por 128 futbolistas varones (integrados en un total de 6 equipos) de competición (entrenan 5 días a la semana en sesiones de 2 horas) de un club deportivo de la Comunidad de Madrid, con edades comprendidas entre 14 y 24 años $(M=17.5, D T=2.5)$. Los jugadores están repartidos en 2 equipos cadetes $(n=43)$ con edades comprendidas entre los 14 y 15 años (pertenecientes a la categoría deportiva Autonómica), 3 equipos juveniles ( $n$ =63) con edades comprendidas entre 16 y 18 años (pertenecientes a las categorías deportiva de Liga Nacional y División de Honor) y 1 equipo de adultos $(n=22)$ con edades comprendidas entre los 19 y 24 años (pertenecientes a la categoría deportiva de Tercera División). En la Tabla 1 se presentan las características de la muestra.

También, participaron en el estudio 6 entrenadores con titulación de Entrenador Nacional (Federación Española de Fútbol) con edades comprendidas entre 26 y 32 años $(M=$ 29.5, DT = 1.7).

Tabla 1. Descriptivos de la variable categoría de edad deportiva.

\begin{tabular}{lcccc}
\hline Categoría de edad & Muestra & Rango edad & Media & D.T. \\
\hline Cadetes & 43 & $15-16$ & 14.50 & 0.51 \\
Juveniles & 67 & $17-18$ & 16.78 & 0.84 \\
Adultos & 22 & $19-24$ & 19.86 & 1.39 \\
\hline Total sujetos & 128 & $14-24$ & 16.55 & 2.03 \\
\hline
\end{tabular}

\section{Variables e Instrumento de medida}

Five-Factor Inventory (NEO-FFI; Costa y McCrae, 1992) en su versión española "inventario NEO reducido de Cinco Factores" (Costa y McCrae, 2008), que proviene del Revised Neo Personality Inventory (NEO-PI-R) o Inventario de Personalidad NEO Revisado, es un cuestionario compuesto por 60 ítems que se agrupan en cinco grandes dimensiones de personalidad: Neuroticismo, Extraversión, Apertura a la Experiencia, Amabilidad y Responsabilidad. Cada ítem se valora en una escala de cinco puntos desde "totalmente en desacuerdo" hasta "totalmente de acuerdo".

Edad, categoría deportiva y entrenador: estas características fueron tomadas directamente de la ficha personal de cada jugador.

Rendimiento deportivo medio: el rendimiento deportivo medio fue evaluado mediante la media sobre 16 observaciones de la actuación de cada deportista en los partidos de liga por parte del entrenador. La puntuación de rendimiento en cada actuación fue computada en función de una escala de 1 a 10 , 
dónde 1 significa el menor rendimiento posible y 10 el máximo. Por tanto, cada entrenador evaluaba el rendimiento deportivo de cada uno de los jugadores de su equipo.

\section{Procedimiento}

La evaluación de los deportistas se realizó de forma grupal una hora antes del horario de inicio de los entrenamientos en una sala de las instalaciones deportivas del club. Antes de realizar la evaluación se les explicó a los jugadores que iban a participar en una investigación sobre Comportamiento y Deporte, y que dichos resultados podían ser comentados, individualmente si lo deseaban, al terminar la temporada.

La participaron fue voluntaria, y dada la minoría de edad de algunos participantes en la investigación, para la cumplimentación de los cuestionarios se ha solicitado la autorización paterna.

La información sobre el rendimiento deportivo se ha recogido de la ficha de cada jugador proporcionada por el entrenador. Los entrenadores valoran el rendimiento deportivo de sus deportistas en una escala de 1 (puntuación mínima) a 10 (puntuación máxima) una vez finalizado cada partido. Esta misma metodología ha sido descrita por diferentes investigadores (García-Naveira y Díaz-Morales, 2010; Lane y Chappell, 2001; Lowther, Lane y Lane, 2002; Thelwell y Maynard, 2003) en deportes como el fútbol, baloncesto y cricket, entre otros. La valoración subjetiva del rendimiento, se basa en que los entrenadores, tras años de experiencia y de observación de jugadores, son capaces de tener una información comportamental relevante de los deportistas (Escudero, 1999; Escudero, Balagué y García-Mas, 2002; Piedmont et al., 1999). Las valoraciones de los entrenadores representan una estimación apropiada de las capacidades de los jugadores, ya que están familiarizados con los criterios de rendimiento (Aptitzsch, 1994; Vanyperen, 1994).

\section{Análisis de datos}

Según los objetivos detallados anteriormente se han utilizado las siguientes técnicas de análisis de datos:

- Métodos de análisis exploratorio, descriptivos básicos de tendencia central (media) y dispersión (desviación típica) para conocer detalladamente los datos de la muestra y las distribuciones de frecuencias.

- Tablas de contingencia y diferencia de medias para relacionar variables nominales y ordinales / continuas, respectivamente.

- Pruebas de normalidad de los datos mediante la Prueba de Kolgomorov-Smirnov.

- Análisis de diferencias de varianza para estudiar las diferencias en personalidad.

- Análisis de correlaciones mediante coeficiente de correlación de Pearson para el establecimiento de posibles relaciones entre los rasgos de personalidad y el rendimiento deportivo.
Análisis de regresión lineal mediante el método de selección de variables hacia atrás (Pardo y Ruiz, 2005) para la valoración de la capacidad predictiva de los rasgos de personalidad con relación al rendimiento.

Para la realización de los análisis de datos se ha utilizado el programa estadístico SPSS v. 19.0

\section{Resultados}

Los resultados se han organizado en dos apartados que se corresponden con los objetivos planteados previamente: análisis de la personalidad en función de la edad y el rendimiento de los deportistas.

\section{Personalidad y categoría de edad deportiva}

Los análisis de normalidad de las cinco dimensiones de personalidad para la muestra global muestra cómo en todos los casos se cumplen los supuestos de normalidad $(p>.05)$.

En cuanto a las diferencias en función de la categoría por edad, los resultados del ANOVA muestra como existen diferencias en los rasgos Apertura a la Experiencia $(F(2,127)=$ 7.403; $p<.001)$ y Responsabilidad $(F(2,127)=3.031 ; p<.05)$. También, se observa una tendencia no significativa en el rasgo Neuroticismo $(F(2,127)=2.296 ; p<.10)$, mientras que no se obtienen diferencias en los rasgos Extraversión y Amabilidad en los grupos anteriormente citados.

Con el objetivo de poder determinar la prueba Post Hoc a utilizar para los tres rasgos donde se han hallado resultados estadísticamente significativos, se utilizó la prueba de homogeneidad de varianzas a través del estadístico de Levene. Dada la ausencia de diferencias estadísticamente significativas en la pruebas, se aplicó la prueba post hoc de Tukey. Respecto al rasgo Apertura de la Experiencia, los jugadores adultos obtienen mayores puntuaciones que los jugadores juveniles $(\phi<.01)$ y cadetes $(p<.001)$. En cuanto al rasgo Responsabilidad, los jugadores adultos obtienen mayores puntuaciones que los jugadores cadetes $(p<.05)$ y juveniles, aunque en este caso no llega a la significación estadística $(p<.10)$. Por último, analizando el rasgo de Neuroticismo, los jugadores juveniles obtienen mayores puntuaciones que los adultos, aunque no se consigue la significación estadística $(p<.10)$. Los resultados se presentan en la Tabla 2.

Partiendo de los criterios propuestos por Cohen (1988), en el rasgo Apertura a la Experiencia, se obtiene un tamaño del efecto grande tanto al comparar deportistas adultos con juveniles $(d=0.80)$ como en deportistas adultos con cadetes $(d=0.97)$, siendo mayor en este segundo caso. En el rasgo Responsabilidad, se obtiene un tamaño de efecto mediano tanto al comparar deportistas adultos con juveniles $(d=0.55)$ como en deportistas adultos con cadetes $(d=0.60)$. Por último, en el rasgo Neuroticismo, se obtiene un tamaño del efecto medio al comparar deportistas adultos con juveniles ( $d=$ $0.59)$. 
Tabla 2. Análisis de las diferencias de personalidad en función de a categoría deportiva por edad de los futbolistas aplicando un ANOVA de un factor.

\begin{tabular}{|c|c|c|c|c|c|c|c|c|c|}
\hline \multirow[b]{2}{*}{$\underline{\text { Rasgos }}$} & \multicolumn{2}{|c|}{ (1) Cadetes $(\mathrm{N}=43)$} & \multicolumn{2}{|c|}{ (2) Juveniles $(\mathrm{N}=63)$} & \multicolumn{5}{|c|}{ (3) Adultos $(\mathrm{N}=22)$} \\
\hline & Media & $D . T$ & Media & $D . T$ & Media & $D . T$ & $F(2,152)$ & Post Hoc Tukey & $d$ \\
\hline Neuroticismo & 17.96 & 7.15 & 18.90 & 5.90 & 15.59 & 5.23 & $2.296(a)$ & $2<3$ (a) & 0.59 \\
\hline Extroversión & 34.49 & 5.74 & 34.83 & 4.64 & 35.50 & 3.83 & 0.308 & - & - \\
\hline Apertura a la Experiencia & 22.98 & 6.08 & 24.46 & 5.49 & 28.73 & 5.75 & $7.403 * * *$ & $\begin{array}{l}3<1 * * * \\
3<2 * *\end{array}$ & $\begin{array}{l}0.97 \\
0.75\end{array}$ \\
\hline Amabilidad & 29.28 & 5.89 & 29.51 & 4.70 & 31.64 & 5.14 & 1.687 & - & - \\
\hline Responsabilidad & 32.40 & 6.64 & 32.97 & 5.48 & 36.09 & 5.71 & $3.031 *$ & $\begin{array}{l}3<1 * \\
3<2 \text { (a) }\end{array}$ & $\begin{array}{l}0.60 \\
0.55\end{array}$ \\
\hline
\end{tabular}

$* p<.05 ; * * p<.01 ; * * * p<.001 ;$ (a) $p<.10$

\section{Personalidad y rendimiento deportivo}

El rendimientos medio evaluado por la escala de 1 a 10 sobre 16 observaciones para todo el grupo fue de 7.2 (DT = 1.4; rango 4.5 a 9.5). Para conocer la dispersión relativa en la variable rendimiento medio se ha calculado el coeficiente de variación (dt/media) de dicha variable para cada participante (García-Naveira, 2010). El resultado obtenido ha informado de un coeficiente de variación medio de 0.12 (varía entre 0 y 1) para toda la muestra. Por lo tanto, parece haber poca variación en las valoraciones individuales sobre el rendimiento hechas por los entrenadores, es decir hay cierta estabilidad del rendimiento entre las observaciones y por lo tanto, la media sobre las 16 observaciones parece ser un buen indicador del rendimiento de cada individuo evaluado.

Para estudiar la relación entre la personalidad y el rendimiento deportivo, se ha realizado análisis correlacionales en función de estas variables utilizando el coeficiente de correlación de Pearson y la técnica de regresión lineal mediante el método de selección de variables hacia atrás como metodología multivariante (Piedmont et al., 1999; Hair, Anderson, Tatham y Black, 1999; Pardo y Ruiz, 2005; Ruiz, 2004; 2006; 2008). Los objetivos de estos análisis son establecer la relación entre las variables estudiadas y el porcentaje de varianza explicada de que disponen los cinco factores de personalidad en relación a las puntuaciones en la valoración del rendimiento realizada por el entrenador. Para ello, en primer lugar se ha utilizado la muestra total de deportistas, para posteriormente realizar un análisis más detallado en función del grupo de edad de los jugadores de fútbol.

Dentro de los diferentes métodos de regresión lineal existentes (Hair et al., 1999; Pardo y Ruiz; 2005), se ha decidido seleccionar el método de selección de variables hacia atrás (Backward o eliminación regresiva; Hair et al., 1999) principalmente por dos motivos. Por una parte, en el presen- te estudio se pretende determinar tanto la capacidad predictiva de modelos que reúnan los cinco factores de personalidad de forma conjunta (modelo completo), como modelos compuestos por un solo factor o conjunto de varios factores (modelos incompletos), pudiendo observar la relevancia y peso específico de cada factor al mantenerse o eliminarse de la regresión. Desde esta perspectiva, Pardo y Ruiz (2005) mencionan como este método "comienza incluyendo en el modelo todas las variables seleccionadas de la lista de independientes y luego procede a eliminarlas una a una" (p. 487). De esta forma, el factor de personalidad que tenga una menor correlación parcial con la valoración del rendimiento, será la primera en ser considerada para su eliminación. Por otra parte, la utilización de este método es debido por su aplicación en estudios precedentes predictivos de personalidad y valoración del rendimiento/resultado deportivo (Ruiz, 2004; 2006; 2008).

Para la muestra total, los resultados indican que el rendimiento deportivo tiene una relación baja y negativa con el rasgo Neuroticismo $(r=-.193 ; p<.05)$ y baja y positiva con los rasgos Apertura a la Experiencia $(r=.181 ; p<.05)$ y Responsabilidad $(r=-.148 ; p<.10)$, aunque en esta última no llega a la significación estadística. Cuanto mayor es la estabilidad emocional, apertura a la experiencia y responsabilidad de los jugadores, mayor es su rendimiento deportivo. También se puede observar que el rasgo Responsabilidad tiene una relación media y negativa con el rasgo Neuroticismo $(r=-.422 ; p<$ .01), y baja y positiva con el rasgo Amabilidad ( $r=.254$; $p<$ .01). Cuanto mayor es la responsabilidad de los jugadores de fútbol, mayor estabilidad emocional y amabilidad. Además, el rasgo Extraversión tiene una relación baja y positiva con el rasgo Apertura a la Experiencia $(r=.239 ; p<.01)$. Cuanto mayor es la extraversión de los deportistas, mayor es la apertura a la experiencia. Los resultados se presentan en la Tabla 3.

Tabla 3. Correlaciones entre las dimensiones del NEO-FFI y el rendimiento deportivo.

\begin{tabular}{lllllll}
\hline Dimensiones & Neuroticismo & Extraversión & Apertura & Amabilidad & Responsabilidad & Rendimiento \\
\hline Neuroticismo & 1 & & & & & \\
Extraversión & -.021 & 1 & & & & \\
Apertura & -.058 & $.239^{* *}$ & 1 & 1 & & \\
Afabilidad & -0.077 & .017 & .034 & $.254^{* *}$ & 1 & $.148(1)$ \\
Responsabilidad & $-0.422^{* *}$ & -.049 & .091 & .105 & 1 \\
Rendimiento & $-.193 *$ & -.026 & $.181 *$ & & \\
\hline$* p<05 ; * *$
\end{tabular}

$* p<.05 ; * * p<.01 ;(1) p<.10$ 
Los análisis multivariantes realizados con la muestra total (Tabla 4) muestran modelos de regresión lineal estadísticamente significativos con una $p<.05$. Incluyendo los cinco rasgos, se obtienen predicciones del $4.2 \%$ de varianza explicada, si bien el modelo ofrece una tendencia a la significación estadística $(p<.10)$. Sin embargo, incluyendo los rasgos Neuroticismo y Apertura a la Experiencia, se obtiene un modelo de regresión con una significación de $p<.05$, siendo la varianza explicada del $5.1 \%$. La ecuación de regresión aplicando el método hacia atrás es la siguiente:

$$
\begin{aligned}
& F=3.084 ; p<.05 ; \text { Rendimiento }=7.092-.033 \text { Neuroticismo }+.032 \\
& \text { Apertura a la Experiencia }
\end{aligned}
$$

Los análisis correlacionales realizados con la categoría por edad deportiva cadete, muestran correlaciones estadísticamente significativas con una $p<.05$ entre la dimensión Neuroticismo y Rendimiento $(r=-.265)$. Los modelos de regre- sión lineal mediante el método hacia atrás (Tabla 4), muestran valores de varianza explicada del $\% \%$, si bien este modelo sólo muestra tendencia significativa $(\mathrm{p}<.10)$. En este caso, las variables introducidas en el modelo serían Extraversión y Neuroticismo. La ecuación de regresión es:

\section{$F=3.084 ; p<.10 ;$ Rendimiento $=8.613-.039$ Neuroticismo -.045 Extraversión}

Respecto al grupo juvenil, no se muestran modelos de regresión lineal estadísticamente significativos, sólo hallando correlaciones con tendencia a la significación estadística $(r=.191 ; p=.067)$ en el rasgo Amabilidad.

Por último, se ha realizado los análisis con el grupo de adultos, hallando correlaciones con tendencia a la significación $(p=.052 ; p<.10)$ de carácter negativo en Neuroticismo con un valor de $r=-.356$. Asimismo, no se hallaron modelos estadísticamente significativos.

\begin{tabular}{|c|c|c|c|c|c|c|c|c|}
\hline \multirow[t]{2}{*}{ Muestra } & & \multirow{2}{*}{$\begin{array}{l}\mathrm{R}^{2} \\
\text { Corregida }\end{array}$} & \multirow[t]{2}{*}{ Sig. } & \multicolumn{4}{|c|}{ Coeficientes no estandarizados } & \multirow[b]{2}{*}{ Sig. } \\
\hline & & & & Modelo & $\mathrm{B}$ & Error típico & $t$ & \\
\hline \multirow[t]{9}{*}{ Muestra global } & $\mathrm{MC}^{1}$ & 0.800 & .067 & Constante & 6.786 & 1.184 & 5.732 & .000 \\
\hline & & & & Neuroticismo & -0.029 & 0.017 & -1.676 & .096 \\
\hline & & & & Extraversión & $-0,017$ & 0.021 & -0.801 & .425 \\
\hline & & & & Apertura & 0.035 & 0.017 & 2.028 & .045 \\
\hline & & & & Afabilidad & 0.017 & 0.020 & 0.862 & .390 \\
\hline & & & & Responsabilidad & 0.008 & 0.019 & 0.410 & .683 \\
\hline & $\overline{\mathrm{MI}^{2}}$ & 0.051 & .014 & Constante & 7.092 & 0.516 & 13.743 & .000 \\
\hline & & & & Neuroticismo & -0.033 & 0.016 & -2.116 & .036 \\
\hline & & & & Apertura & 0.032 & 0.016 & 1.967 & .051 \\
\hline \multirow[t]{3}{*}{ Muestra cadete } & $\mathrm{MI}^{2}$ & 0.90 & .057 & Constante & 8.613 & 1.001 & 8.604 & .000 \\
\hline & & & & Neuroticismo & -0.039 & 0.021 & -0.1866 & .069 \\
\hline & & & & Extraversión & -0.045 & 0.026 & -1.714 & .094 \\
\hline
\end{tabular}

Tabla 4.- Análisis de regresión lineal múltiple mediante el método hacia atrás para la muestra total de futbolistas y la categoría cadete.

$\mathrm{MC}^{1}=$ Modelo completo

$\mathrm{MI}^{2}=$ Modelo incompleto

Partiendo de estos datos, se ha incorporado un análisis más específico en función del grupo de edad de los jugadores. Como ya comentaba Ruiz (2004, 2006, 2008), es necesario establecer análisis específicos entre las relaciones de personalidad y rendimiento, considerando principalmente la variable edad y sexo. En una misma categoría por edad deportiva pueden existir importantes diferencias debidas a la edad (por ejemplo, en la categoría juvenil pueden existir hasta 3 años de diferencia entre los jugadores) o que un jugador de una edad esté jugando en una mayor categoría por edad (por ejemplo, un jugador de 15 años que juega en la categoría juvenil), y madurativamente esto puede afectar en las posibles diferencias físicas, técnicas, tácticas y psicológicas. Por ello, se decidió realizar análisis correlacionales y establecer modelos de regresión en función de grupos de edad, dividiendo los análisis en grupos de 14, 15, 16, 17, 18 años y más de 18 años.

Considerando a los futbolistas de 14 años $(n=22)$, los análisis correccionales muestran correlaciones con tendencia a la significación estadística $(p=.088 ; p<.010)$ en el rasgo Neuroticismo, siendo la correlación de $r=-.372$. Asimismo, se observaron modelos de regresión estadísticamente significativos con una $p<.05$ y un porcentaje de varianza explicada del 24.3\% (Tabla 5). Los rasgos introducidos en el modelo fueron Neuroticismo y Responsabilidad. La ecuación de regresión es:

\section{$F=4.361 ; p<.05 ;$ Rendimiento $=8.903-.063$ Neuroticismo -.058 Responsabilidad}

Respecto a los futbolistas de 15 años $(n=23)$, sólo se observan correlaciones negativas con tendencia a la significación en el rasgo Neuroticismo ( $r=-.343 ; p=.055 ; p<.10)$, no hallando modelos de regresión estadísticamente significativos.

Considerando los futbolistas de 16 años $(n=23)$, se observan correlaciones negativas estadísticamente significativas en el rasgo Neuroticismo ( $r=-.353 ; p=.049 ; p<.05)$. Con esta misma variable, se observan modelos de regresión con tendencia a la significación estadística, sólo hallando una varianza explicada del 8.3\% (Tabla 5). La recta de regresión es: 


\section{$F=2.984 ; p<.10 ;$ Rendimiento $=8.524-0.48$ Neuroticismo}

Respecto a los jugadores de 17 de años $(n=23)$, sólo se obtienen correlaciones con tendencia a la significación estadística de forma negativa en el rasgo Responsabilidad ( $p=.094 ; p<.10 ; r=-.284)$, no obteniéndose modelos de regresión estadísticamente significativos.

Por otro lado, con los jugadores de 18 años $(n=16)$, sólo se obtienen correlaciones positivas con tendencia a la significación en el rasgo Extraversión ( $p=.075 ; p<.10 ; r=.377$ ), no hallándose modelos de regresión estadísticamente significativos.
Por último, y siguiendo con la tendencia de resultados de los futbolistas de 14 a 16 años de edad, los futbolistas de más de 18 años $(n=21)$ obtienen correlaciones estadísticamente significativas negativas con una $p<.05(p=.022)$ en el rasgo Neuroticismo ( $r=-.444)$. A su vez, se obtienen modelos de regresión estadísticamente significativos con una $p<.05$ incluyendo los factores Neuroticismo y Extraversión, proporcionando una varianza explicada del $25.6 \%$ (Tabla 5). La ecuación de regresión es:

\section{$F=4.433 ; p<.05 ;$ Rendimiento $=11.362-.059$ Neuroticismo. .054 Extraversión.}

Tabla 5. Análisis de regresión mediante el método hacia atrás para los futbolistas de 14, 16 y más de 18 años de edad.

\begin{tabular}{|c|c|c|c|c|c|c|c|c|}
\hline \multirow[t]{2}{*}{ Muestra } & & \multirow{2}{*}{$\begin{array}{l}\mathrm{R}^{2} \\
\text { Corregida }\end{array}$} & \multirow[t]{2}{*}{ Sig. } & \multicolumn{5}{|c|}{ Coeficientes no estandarizados } \\
\hline & & & & Modelo & B & Error típico & $t$ & Sig. \\
\hline \multirow[t]{3}{*}{ Futbolistas de 14 años } & $\mathrm{MI}^{2}$ & 0.243 & .028 & Constante & 8.903 & 1.071 & 8.309 & .000 \\
\hline & & & & Neuroticismo & -0.063 & 0.024 & -2.644 & .016 \\
\hline & & & & Responsabilidad & -0058 & 0.026 & -2.211 & .039 \\
\hline \multirow[t]{2}{*}{ Futbolistas de 16 años } & $\mathrm{MI}^{2}$ & 0.083 & .099 & Constante & 8.524 & 0.603 & 14.132 & .000 \\
\hline & & & & Neuroticismo & -0.048 & 0.028 & -1.727 & .099 \\
\hline \multirow[t]{3}{*}{ Futbolistas con más de 18 años } & $\mathrm{MI}^{2}$ & 0.256 & .027 & Constante & 11.362 & 1.152 & 9.864 & .000 \\
\hline & & & & Neuroticismo & -0.059 & 0.021 & -2.767 & .013 \\
\hline & & & & Extraversión & -0.054 & 0.028 & -1.888 & .075 \\
\hline
\end{tabular}

$\mathrm{MI}^{2}=$ Modelo incompleto

\section{Discusión}

Con el objetivo de contribuir al estudio de la personalidad en el ámbito deportivo, se han investigado la relación entre personalidad, edad y rendimiento de los deportistas. Por un lado, los resultados muestran como existen diferencias en función de la categoría por edad de los deportistas, en la que los jugadores adultos obtienen mayores puntuaciones en el rasgo Estabilidad Emocional que los jugadores juveniles. También, los jugadores adultos tienen un mayor rasgo de Apertura a la Experiencia y Responsabilidad que los jugadores cadetes y juveniles. Por otro lado, los resultados indican que determinados características de personalidad se relacionan con un mayor rendimiento deportivo. Así, para la muestra total de deportistas, los rasgos Responsabilidad, la Apertura a la Experiencia y la Estabilidad Emocional se relacionan de forma positiva con el rendimiento deportivo. En este caso se obtiene modelos de regresión significativos $(p<.05)$ con una capacidad predictiva del $5.1 \%$ introduciendo las variables Neuroticismo y Apertura a la Experiencia. Respecto a la categoría por edad, sólo se muestran modelos de regresión con tendencia a la significación estadística $(p<.10)$ en la categoría cadete. Sin embargo, incluyendo en los modelos de regresión la variable grupo de edad del futbolista, se obtienen modelos de regresión lineal significativos de forma selectiva para los futbolistas de 14 años y mayores de 18 años $(p<.05)$, llegando a predicciones del $24.3 \%$ y $25.6 \%$ respectivamente. Las variables introducidas en el modelo de regresión para los futbolistas de 14 años son Neuroticismo y Reponsabilidad, y Neuroticismo y Extraversión para los futbolistas mayores de 18 años. A continuación se discuten dichos resultados.

\section{Personalidad y diferencias de edad deportiva}

El objetivo del presente apartado era el de analizar las diferencias en personalidad de los jugadores de fútbol en función de la categoría de edad deportiva. Los resultados obtenidos cumplen parcialmente la hipótesis 1 (los deportistas adultos obtienen mayores puntuaciones en las dimensiones de Responsabilidad, Amabilidad y Apertura a la Experiencia que los deportistas de menor categoría (cadetes y juveniles), mientras que estos tienen mayores puntuaciones en Neuroticismo que los adultos). Estos datos están a favor de los resultados obtenidos por Ruiz (2005) y GarcíaNaveira et al. (2011), donde los judokas y jugadores de fútbol adultos tienen mayores puntuaciones en los rasgos Apertura a la Experiencia, Responsabilidad y Estabilidad Emocional que los de menor categoría de edad. A diferencia de estos estudios, no se han obtenido diferencias en el rasgo Amabilidad.

Además de estas diferencias, destacar la jerarquización del tamaño del efecto de estos rasgos (según los criterios de Cohen, 1988) en los que la Apertura a la Experiencia estaría a un nivel superior con un efecto grande, frente al Neuroticismo y la Responsabilidad con un efecto mediano. Resultados similares fueron obtenidos por García-Naveira et al. (2011). Estos datos podrían suponer:

La relevancia de la amplitud y variedad de los intereses, así como la intensidad de la vida emotiva de los deportistas adultos frente a los más jóvenes (rasgo Apertura a la Experiencia). La experiencia deportiva (aprendizajes, entrenamientos, competiciones...) puede que influya sobre 
la apertura del deportista hacia nuevas experiencias, acontecimientos y búsqueda de sensaciones.

La mayor inestabilidad emocional, ansiedad, ira, hostilidad y depresión de los deportistas jóvenes frente a los adultos (rasgo Neuroticismo). La exposición del deportista ante situaciones potencialmente estresantes que se dan en el deporte de alto rendimiento (p. ej., exigencia deportiva, marcador en contra, presión del público, etc.), a las que tiene que hacer frente para rendir, puede que favorezca al desarrollo de una mayor estabilidad emocional y/o aprendizaje de estrategias de afrontamiento (p. ej., relajación, auto-instrucciones, etc.).

- La mayor responsabilidad de los adultos frente a los más jóvenes (rasgo Responsabilidad). El cumplimiento de normas y rutinas y el desempeño de roles y funciones del deportista en el deporte de alto rendimiento puede que forme personas más disciplinadas, ordenadas y motivadas hacia el logro.

Otro dato importante es la ausencia de diferencias estadísticamente significativas en el rasgo Extroversión. A diferencia de otras características de personalidad, el papel que puede jugar la extroversión ya desde categorías jóvenes puede ser relevante, dado que el fútbol es un deporte de equipo, con una alta tasa de interacciones deportista-deportista y deportista-entrenador, entre otros. Este aspecto podría constituirse como una variable relevante en el desarrollo de los entrenamientos, las competiciones y el contexto deportivo en general (García-Naveira et al., 2011; Piedmont et al., 1999).

Esta situación nos plantea una cuestión ya planteada por anteriores autores referidas a la hipótesis de selección vs. hipótesis de desarrollo (Bakker, Whiting y Van Der Drug, 1992; Wann, 1997). Las características del estudio desarrollado (diseño transversal) no nos permite determinar cuál de estas dos hipótesis se está poniendo en juego, por lo que se sugiere realizar en futuros trabajos un estudio longitudinal de la personalidad de los deportistas. A pesar de ello, en la línea de las conclusiones de García-Naveira et al. (2011), a la vista del desarrollo teórico e investigador en personalidad, tanto la "selección" como el "desarrollo" en la práctica de un deporte influyen conjuntamente en el perfil de personalidad del individuo. El paso del joven deportista por experiencias y exigencias deportivas, puede incrementar los niveles de determinadas características de personalidad y estabilizarse en la edad adulta, si bien los factores evolutivos y madurativos pueden estar interviniendo en mayor o menor medida durante este proceso.

\section{Personalidad y rendimiento deportivo}

El objetivo de este apartado era analizar la relación entre la personalidad y el rendimiento deportivo. Partiendo de los datos obtenidos para la muestra total de deportistas, se cumple parcialmente la hipótesis 2 (las dimensiones de Responsabilidad y Extraversión se asocian positivamente con el rendimiento deportivo, mientras que el Neuroticismo lo hace de forma negativa). Los resultados apoyan tanto a los trabajos que definen a los deportistas de alto rendimiento o de élite como responsables (García-Naveira et al., 2011; Kane, 1964; Olgilvie, 1968; Ruiz, 2001, 2004; Tutko y Olgilvie, 1969) y estables emocionalmente (Daino, 1985; Gabler 1976; García-Naveira et al., 2011; Kane, 1964; Kirkcaldy, 1982; Olgilvie, 1968; Ruiz, 2001, 2004, 2012; Tutko y Olgilvie 1969) como a las investigaciones que relacionan de forma positiva al rendimiento deportivo con la responsabilidad (Piedmont et al., 1999; Ruiz, 2006) y la estabilidad emocional (Karp, 2000; Piedmont et al., 1999). Por el contrario, no se obtiene relación entre el rasgo Extraversión y el rendimiento deportivo. Además, el rasgo Apertura a la Experiencia correlaciona positivamente con el rendimiento deportivo, aspecto relacionado al resultado obtenido por Ruiz (2012) al comparar los deportistas de combate con la población normal de referencia.

Coincidiendo con García-Naveira et al. (2011), los rasgos de Responsabilidad y Estabilidad Emocional cobran un especial interés en el deporte de competición. Por un lado, el deportista para hacer frente a las demandas del alto rendimiento (entrenamientos, horarios, competiciones, desplazamientos, etc.) requieren orden, competencia, sentido del deber, orientación al logro y auto-disciplina. Por otro lado, es de destacar que el deporte de competición puede exponer al deportista ante situaciones altamente estresantes (p. ej., exigencias del juego, marcador en contra, presión del público, etc.), por lo que la estabilidad emocional sería necesaria para obtener el máximo rendimiento.

Además de su relación con el rendimiento deportivo, ambos rasgos (Responsabilidad y Neuroticismo) son relevantes al estar relacionados entre sí de forma negativa, por lo que sería importante contribuir (p.ej., programas de intervención) a que los deportistas desarrollen ambos aspectos. En esta línea, por un lado, crear un contexto de trabajo con normas, orden y disciplina puede ofrecer un marco de referencia que estabilice emocionalmente al deportista, y por otro lado, el aprendizaje de técnicas de autorregulación emocional (p.ej., relajación, autoinstrucciones, etc.) puede que favorezca a la persona a que esté más centrada en las tareas a realizar y en su gestión personal.

También, el rasgo de Responsabilidad se relaciona de forma positiva con el rasgo Amabilidad. En un deporte de equipo como el fútbol, además de la autogestión individual, existen tareas a desarrollar a nivel grupal (p. ej., recogida del material, coordinación de acciones defensivas, etc.) que puede que esté relacionado con el "buen vínculo" que tenga el deportista con los demás. Por tanto, la generación de ambientes de trabajo orientados a las relaciones puede que favorezca la responsabilidad de los deportistas.

En cuanto al rasgo Extraversión, los resultados no apoyan a los trabajos que definen al deportista de alto nivel como extrovertido (Bushan y Agarwal, 1978; Daino, 1985; Egloff y Gruhn, 1996; Foster, 1977; Gabler, 1976; GarcíaNaveira et al., 2011; Kane, 1964; Kirkcaldy, 1982; Novotny y Petrak, 1983; Olgilvie 1968; Ruiz, 2004; Tutko y Olgilvie, 
1969) ni a las investigaciones que relacionan de forma positiva a la extraversión con el rendimiento deportivo (Graydon y Murphy, 1995; Ruiz, 2007, 2012).

A pesar que el rasgo Extraversión es considerado por algunos autores como el predictor más importante de la actividad física (Rhodes y Smith, 2006), puede que cumpla una labor indirecta sobre el rendimiento (Piedmont et al., 1999). En este sentido, García-Naveira et al. (2011) indican que es posible que la Extraversión sea un pre-requisito para la práctica de los deportes de equipo de competición, en la que los deportistas de mayor nivel son más extravertidos que los de menor nivel. Este aspecto podría tener al menos dos posibles interpretaciones. Por un lado, en los deportes de equipo de competición las relaciones sociales, la búsqueda de emociones, actividad, etc., son importantes como vínculo entre las personas, y tal vez se relacione con otras variables grupales como la comunicación y cohesión (vertiente social). A pesar de este aspecto, la extraversión no se relacionaría directamente con la propia ejecución de la tarea en deportes de equipo.

Por otro lado, Smith y Rhodes (2006) concluyen que los deportistas de deportes de equipo son más extravertidos que los deportistas de deportes individuales. En este sentido, en deportes individuales de competición como el tenis de mesa (Graydon y Murphy, 1995) y el judo (Ruiz, 2006) si se ha obtenido una relación positiva entre el resultado deportivo y la Extraversión, por lo que esta variable puede que tenga un especial interés como facilitación social ante la presencia de público en esta modalidad deportiva.

En cuanto al rasgo de Apertura a la Experiencia, los datos podrían indicar la relevancia de la amplitud y variedad de los intereses, así como la intensidad de la vida emotiva sobre el rendimiento deportivo. Sería importante determinar en posteriores estudios este aspecto, ya que este rasgo es generalmente llamado como apertura a la experiencia (Digman, 1990), aunque también es definido por otros teóricos como intelecto (entendimiento y potencia cognoscitiva racional) (Goldberg, 1993), aspectos que tal vez se asociarían al aprendizaje, la comprensión táctica y la toma de decisiones. Además, el rasgo Apertura a la Experiencia podría representar un aspecto de la cognición relacionada con la búsqueda de sensaciones (Diehm y Armatas, 2004) y orientación hacia nuevas experiencias (Ruiz, 2012), por lo que el deportista encontraría dicha estimulación en las acciones del propio rendimiento.

Además, el rasgo Apertura a la Experiencia se relaciona de forma positiva con la Extraversión. Este aspecto podría suponer que existe una doble tendencia a buscar estimulación a nivel cognitivo por parte del deportista, una de carácter social y otra orientada a los acontecimientos externos.

Una vez realizados los análisis correlacionales de la muestra total utilizada, se puede observar cómo se obtienen modelos de regresión estadísticamente significativos $(p<.05)$, si bien el porcentaje de varianza explicada es reducida $(5.1 \%)$.
Ante este hecho y siguiendo las recomendaciones de Ruiz (2005; 2006; 2008) de controlar las variables edad y sexo, los análisis de categorías por edad sólo muestran modelos con tendencia a la significación estadística $(\mathrm{p}<.10)$ considerando la categoría cadete. Asimismo, se observa de forma evidente como existen correlaciones estadísticamente significativas o con tendencia a la significación de carácter negativo entre la dimensión Neuroticismo y Rendimiento en las categorías cadete y adultos, no mostrándose esta tendencia en el grupo juvenil.

Estos datos sugirieron realizar un análisis aún más específico, considerando exclusivamente el grupo de edad de los futbolistas. Los análisis más analíticos y moleculares mostraron de forma clara que los futbolistas de 14, 15, 16 y mayores de 18 años, obtuvieron correlaciones negativas estadísticamente significativas o con tendencia a la significación entre el Rendimiento y el rasgo Neuroticismo.

En cuanto a los modelos de regresión, se observan modelos de regresión lineal estadísticamente significativos $(p<.05)$ tanto en los futbolistas cadetes de 14 años como en los jugadores de más de 18 años, llegando respectivamente a valores del $24.3 \%$ y $25.6 \%$ de varianza explicada. Asimismo, el grupo de 16 años obtiene un menor valor explicativo al sólo llegar al $8.3 \%$ con modelos de regresión con tendencia significativa $(p<.10)$.

Estos resultados muestran la importancia de la variable Neuroticismo como rasgo que correlaciona de forma inversa con el rendimiento utilizando el sistema de valoración del rendimiento en el presente estudio. Asimismo, también se observa como el papel de los rasgos Responsabilidad y Extraversión quedan subordinados al aparecer sólo en modelos de regresión en el grupo de 14 años y más de 18 años respectivamente, $\mathrm{y}$ ambos a su vez de forma inversa y negativa con el Rendimiento.

Como señala Morgan (1980, citado en Vanden Auweele et al., 1993) hay que considerar cualquier variable psicológica como relevante si la capacidad predictiva explica al menos el $20 \%$ del rendimiento. Por este motivo, aunque sea de forma selectiva, para los grupos de edad de 14 y más de 18 años, el rasgo Neuroticismo toma un valor prioritario, mientras los rasgos Responsabilidad y Extroversión ocupan un segundo lugar, mostrando resultados diferenciales con estudios precedentes (Piedmont et al., 1999; Ruiz, 2004; 2006; 2008).

Cabe señalar que los resultados de una asociación negativa de los rasgos Responsabilidad (deportistas de 14 años) y Extraversión (jugadores mayores de 18 años) con el rendimiento deportivo a priori son contra intuitivos. A pesar de ello, por un lado, una posible respuesta se puede encontrar en las características del propio juego (p.ej., menor búsqueda de resultados y rigidez en el sistema táctico de juego) que todavía se puede dar hasta la categoría cadete de primer año (14 años), en el que una menor responsabilidad se relaciona con un mayor rendimiento ("mayor libertad en el juego"). Por otro, un contexto de alto rendimiento, centrado en la búsqueda de resultados, con máxima exigencia deportiva y que además es selectivo (solo algunos progresan de categoría 
deportiva o equipo) en adultos (mayores de 18 años) puede generar cierto retraimiento social, que al menos puede favorecer el rendimiento individual del deportista ("estar más centrado en uno mismo").

Asimismo, se debe de ser prudentes con la generalización de los resultados obtenidos al aplicar los modelos de regresión lineal, dado que en algunos subgrupos de edad (jugadores con 18 años) el ratio de variable (dimensiones de personalidad) por caso es algo mayor de 3 (3.2), si bien el resto de casos la proporción es mayor de 4 (entre 4.2 y 4.6), debiendo en posteriores estudios incrementar la muestra con el objetivo de garantizar una mayor grado de generalización de los datos.

En resumen, en la actualidad, el estudio de la personalidad en el contexto deportivo continúa en un período de esclarecimientos de resultados, aunque en los últimos 10 años se han ido logrando importantes avances y resultados. Estos resultados, por un lado, sugieren una "hipótesis integral" del análisis de la personalidad en función de la edad de los deportistas (hipótesis de desarrollo + hipótesis de selección). La relación entre la personalidad y el deporte podría describirse como un darwinismo deportivo (la supervivencia del más apto), sin embargo, esto no excluye la posibilidad de que la práctica deportiva pueda mejorar el desarrollo de la personalidad. Por otro lado, los datos apuntan a una "perspectiva crédula" en los que ciertos rasgos de personalidad favorecen o disminuye el rendimiento deportivo.

En concreto, para la muestra total de deportistas, en el presente estudio destacan los rasgos Apertura a la Experiencia, Responsabilidad y Neuroticismo. El rasgo Apertura a la Experiencia varía en función de la edad (mayor en adultos frente a los más jóvenes), con un tamaño del efecto grande y se relaciona positivamente con el rendimiento deportivo. Además, el rasgo Responsabilidad varía en función de la edad (mayor en adultos frente a los más jóvenes), con un tamaño del efecto mediano y se relaciona positivamente con el rendimiento deportivo. También, el rasgo Neuroticismo varía en función de la edad (mayor en los más jóvenes frente a los adultos), con un tamaño del efecto medio y se relaciona negativamente con el rendimiento deportivo. Por el contrario, los rasgos Extraversión y Amabilidad no varían en función de la edad y no se relacionan con el rendimiento deportivo.

En cuanto al rendimiento, a pesar de esta valoración, hay que tener presente que estas relaciones son bajas. Esto puede ser debido a que el rendimiento y resultado de un deportista es multifactorial (Williams y James, 2001) y no tienen

\section{Referencias}

Apitzsch, E. (1994). La personalidad del jugador de fútbol de élite. Revista de Psicología del Deporte, 6, 89-98.

Arbinaga, F. y Caracuel, J. C. (2008). Rasgos de personalidad en fisicoculturistas y relaciones con variables antropométricas y conductas deportivas. Revista de Psicología del Deporte, 17 (1), 85-102.

Bakker, F., Whiting, H. T. y Van Der Brug, H. (1992). Psicología del deporte. Conceptos y aplicaciones. Madrid: Morata. una relación de causa efecto (Weinberg y Gould, 2010). También, para atrapar la complejidad de la pericia en los diferentes deportes se requiere un enfoque multitarea (Ruiz, Sánchez, Durán y Jiménez, 2006). Además, desde una perspectiva de análisis multivariante, se plantea la necesidad de controlar no sólo la variable categoría por edad deportiva, sino también el grupo de edad dentro de cada categoría debido a los cambios madurativos y psicobiológicos que se producen entre los cadetes (14-15 años), los juveniles (16-18 años) y los adultos (mayores de 18 años). En este sentido, el rasgo Neuroticismo cobra un especial protagonismo como un posible "inhibidor" del rendimiento deportivo en los jugadores de fútbol.

Como limitaciones metodológicas del estudio, principalmente se pueden señalar tres: 1) limitaciones de la aproximación trasversal, que no permite concluir si los rasgos de personalidad cambian con la práctica deportiva o si, por el contrario, el proceso selectivo propio del progreso en la carrera deportiva elimina de las categorías superiores a aquellos individuos psicológicamente menos aptos, 2) aunque su uso es frecuente, las medidas subjetivas de rendimiento (evaluación por parte del entrenador o deportista) plantean ciertos problemas de validez. Debe considerarse la posibilidad de que los rasgos de personalidad del deportista no sólo afecten al rendimiento objetivo, sino también a la percepción que los entrenadores tengan de ese rendimiento y 3) la necesidad de tener una muestra lo suficientemente amplia para realizar un análisis multivariante en función de la edad de los deportistas al estudiar la relación entre los rasgos de personalidad y el rendimiento deportivo.

Partiendo de estos y otros aspectos que se han presentando en este trabajo, como perspectivas futuras se recomienda realizar estudios longitudinales de la evolución de la personalidad en función de la edad de los deportistas, evaluar el rendimiento tanto con medidas objetivas como subjetivas, aplicar una metodología multivariante en el estudio del rendimiento, analizar el rendimiento en función de la edad de los deportistas, abordar otras variables propias (capacidad física, técnico-táctica, competencias psicológicas, etc.) y ajenas del deportista (acciones de los rivales, del entrenador, etc.) para tener una visión más amplia del mismo y su relación con el rendimiento deportivo, utilizar la evaluación de la personalidad como un complemento a los procedimientos habituales (ojeadores, médicos, etc.) en la detección de talentos y selección de deportistas y realizar intervenciones para potenciar los rasgos asociados con un mayor rendimiento deportivo.

Bara, M., Scipiao L. y Guillén, F. (2005). La personalidad de deportistas y no deportistas: jexisten realmente diferencias? Comunicación presentada en el X Congreso Nacional Andaluz de Psicología del Deporte. Málaga. Bermúdez, J. (1995). Cuestionario "Big Five". Madrid: TEA Ediciones.

Bushan, S. y Agarwal, V. (1978). Personality characteristics of high an low achieving Indian sports persons. International Journal of Sport Psychology, 9 (3), 193-198. 
Casis, L. y Casis, L. y Zumalabe, J.M․ (2008). Fisiología y psicología de la actividad física y el deporte. Barcelona: Elsevier.

Cattell, R.B., Cattell, A.K. y Cattell, H.E.P. (1993). Sixteen personality factor questionnaire (Fifth Edition). Champaign. IL: Institute for Personality and Ability Testing.

Cohen, J. (1988). Statistical power analysis for the behavioral sciences (2nd). Erlbaum, Hillsdale, NJ.

Colegio Oficial de Psicólogos de España (2011). Inventario de personalidad NEO revisado. Madrid: COP

Costa, P. T. y McCrae, R. R. (1992). Neo-PI-R: Revised NEO Personality Inventory (NEO-PI-R). Odessa, F.L: Psychological Assessment Resources.

Costa, P.T. y McCrae, R.R. (2008). NEO PI-R Inventario de Personalidad NEO Revisado. TEA Ediciones

Cox, R. H. (2009). Psicología del deporte: conceptos y sus aplicaciones (6 $6^{\mathrm{a}}$ ed.). Madrid: Editorial Médica Panamericana.

Daino, A. (1985). Personality traits of adolescent tennis players. Internacional Journal of Sport Psychology, 16, 120-125.

Diehm, R. y Armatas, C. (2004). Surfing: an avenue for socially aceptable risk-taking, satisfying needs for sensation seeking and experience seeking. Personality and Individual Differences, 36, 663-677.

Digman, J. M. (1990). Personality structure: Emergence of the five-factor model. Annual Review of Psychology, 41, 417-440.

Egloff, B. y Gruhn, A.J. (1996). Personality and endurance sports. Personality and Individual Differences, 21(2), 223-229.

Elman, W. y McKelvie, S. (2003). Narcissism in football players: Stereotype o reality. Athletic Insight (On-Line), 5. Recuperado el 23 de Enero de 2011, de http://www.athleticinsight.com/Vol5Iss1/Narcissism.htm

Escudero, J. (1999). Uso y conocimiento de variables psicológicas y evaluación de comportamientos deportivos de jugadores por parte de los entrenadores de baloncesto orientados al rendimiento. Tesis doctoral no publicada. Departamento de Psicología. Universitat Illes Balears.

Escudero, J. Balagué, G., y García-Mas, A. (2002). Comportamientos que influyen en el rendimiento deportivo de jugadores de baloncesto desde el punto de vista de los entrenadores. Psicothema, 14 (1), 34-38.

Eysenck, H. J. y Eysenck, S. B. (1975). Manual of the Eysenck Personality Questionnaire. London: Hodder and Stoughton

Fenández, J.L., Fernández, I., y Mielgo, M. (1999). PY-BTPD-S. Batería de tests psicológicos para deportistas-Salamanca. Madrid. Psymtéc.

Foster, W. (1977). A discriminant analysis of selected personality variables among successful and unsuccessful male high school athletes. International Journal of Sport Psychology, 8, 119-127.

Gabler, H. (1976). Desarrollo de los rasgos de personalidad en deportistas de alto nivel. Sportwissenschaft, 6, 247-276.

García-Naveira, A. (2010). Diferencias individuales en Estilos de Personalidady rendimiento en deportistas. Tesis Doctoral. Facultad de Psicología. Universidad Complutense de Madrid. Recuperado el 10 de marzo de 2011, de http://eprints.ucm.es/9859/1/T31529.pdf

García-Naveira, A. y Díaz-Morales, J. F. (2010). Relación entre optimismo/ pesimismo disposicional, rendimiento y edad en jugadores de fútbol de competición. Revista de Iberoamericana de Psicología del Ejercicio y el Deporte, 5 (1), 45-60.

García-Naveira, A., Ruiz, R. y Pujals, C. (2011). Diferencias en personalidad en función de la práctica o no deportiva, nivel de competición y categoría por edad en jugadores de fútbol desde el modelo de Costa y McCrae. Revista de Psicología del Deporte, 20 (1), 29-44.

Goldberg, L.R. (1993). The structure of phenotypic personality traits. American Psychology, 48, 26-60.

Graydon, J. y Murphy, T. (1995). The effect of personality on social facilitation whilst performing a sports related task. Personality and Individual Differences, 19 (2), 265-267.

Hair, J. F., Anderson, R. E., Tatham, R. L. y Black, W. C. (1999). Análisis multivariante ( $5^{\text {a }}$ edición). Madrid: Pearson Educación.

Hoyt, A., Rhodes, R., Hausenblas, H. y Giacobbi, P. (2009). Integrating fivefactor model facet-level traits with the theory of planned behavior and exercise. Psychology of Sport and Exercise, 10, 565-572.

Kane, J. (1964). Personality and physical ability. Comunicación presentada en el International Congress Sport Sciences Proceedings. Tokio.

Karp, P. E. (2000). Personality assessment and the prediction of success and achievement in professional hockey. Dissertation Abstracts International: Section B: The Sciences and Engineering, 61 (6-B), 3315.
Kirkcaldy, B.D. (1982). Personality profiles at various levels of athletics participation. Personality and Individual Differences, 3 (3), 321-326.

Lane, A.M. y Chappell, R.H. (2001). Mood and performance relationships at the World Student Games basketball competition. Journal of Sport Behavior, 24, 182-196.

Lowther, J., Lane, A. y Lane, H. (2002). Self-efficacy and psychological skills during The Amputee Soccer World Cup. Atbletic Insight (On-Line), 4. Recuperado el 23 de Enero de 2011, de http://www.athleticinsight.com/Vol4Iss2/SoccerSelfEfficacy.htm.

Marrero, G., Martin-Albo, J. y Nuñez, J. L. (2000). Perfil de personalidad de tenista. Revista de Psicología del Deporte, 9 (1-2), 21-36.

Marín, A.A., Piñar, M.I., Brox, V., Miranda, T.M., Suárez, E., Alarcón, F., y Cárdenas, D. (2012). Características de la personalidad de jóvenes jugadores de baloncesto (15-under). Cuadernos de Psicologia del Deporte, 12(1), 135-138.

McKelvie, S. J., Lemieux, P. y Stout, D. (2003). Extraversion and neuroticism in contact athletes, no contact athletes and non-athletes: A research note. Atbletic Insight (On-Line), 5. Recuperado de http://www.athleticinsight.com/Vol5Iss3/ExtraversionNeuroticism.htm.

Morgan, W.P (1980). The Trait Psychology Controversy. Research Quarterly for Exercise and Sport, 51 (1), 50-76.

Novotny, L. y Petrak, B. (1983). Characteristics of juniors and schoolboy ice hockey players. International Journal of Sport Psychology, 14 (1), 15-26.

Ogilvie, B. (1968). Psychological consistencies within the personality of high-level competitors. Journal of the American Medical Association, 205 (11), 780-786.

Pardo, A. y Ruiz, M.A. (2005). Análisis de datos con SPSS 13 base. Madrid: McGraw-Hill.

Piedmont, R. L., David, C. H. y Blanco, S. (1999). Predicting athletic performance using the five-factor model of personality. Personality and Individual Differences 27, 769-777.

Reyes, M., Raimundi, M.J., y Gómez, L. (2012). Cuadernos de Psicología del Deporte, 12(1), 9-16.

Rhodes, R. y Smith, N. (2006). Personality correlatos of physical activity: a review and meta-análisis. British Journal of Sports Medicine, 40 (12), 958965.

Rodríguez, M. (2003). Diagnóstico de personalidad en deporte de competición: fútbol. Tesis Doctoral. Facultad de Medicina. Universidad Las Palmas de Gran Canaria.

Ruiz, R. (2001). La profesión del deportista desde la perspectiva de los rasgos de personalidad. Infocoes, 1 (1), 49-72.

Ruiz, R. (2004). Análisis de las características psicológicas, deportivas y sociales en el deporte del judo a nivel competitivo: Una propuesta teórica y metodológica para la predicción del rendimiento. Tesis doctoral no publicada. Universidad Autónoma de Madrid. Madrid.

Ruiz, R. (2005). Análisis de diferencias de personalidad en el deporte del judo a nivel competitivo en función de la variable sexo y categoría de edad deportiva. Cuadernos de Psicología del Deporte, 5 (1 y 2), 29-48.

Ruiz, R. (2006). Predicción del resultado deportivo en judokas cadetes de competición aplicando las dimensiones del cuestionario de personalidad BFQ. Revista Iberoamericana de Psicología del Ejercicio y el Deporte, 1 (2), 69 88.

Ruiz, R. (2008). Aportaciones del análisis subdimensional del cuestionario de personalidad BFQ para la predicción del rendimiento en judokas jóvenes de competición. Cuadernos de Psicología del Deporte, 8 (1), 5-29.

Ruiz., R. (2012). Relaciones entre características de personalidad y estados de ánimo: un estudio con deportistas de combate universitarios. Revista Iberoamericana de Psicología del Ejercicio y el Deporte, 7 (1), 89-112.

Ruiz, L. M., Sánchez, M., Durán, J. y Jiménez, C. (2006). Los expertos en el deporte: su estudio y análisis desde una perspectiva psicológica. Anales de Psicología, 23 (2), 300-306.

Silva, J. M. III (1984). Personality and sport performance: controversy and challenge. En J. M. Silva y R. S. Weinberg (Eds.), Psychological foundations of sport (pp.43-74). Human Kinetics.

Tattersfield, C.R. (1971). Competitive sport and personality development. Unpublished doctoral dissertation, University of Durham, NC.

Thewell, R. C. y Maynard, I. W. (2003). The effects of mental skills package on "repeatable good performance" in cricketers. Psychology of Sport and Exercise, 4(4), 377-396. 
Tutko, T. y Ogilvie, B. (1969). The athletic motivational inventory. San Jose: Institute for the Study of Athletic Motivation.

Valdés, H.M. (1998). Personalidady Deporte. Barcelona: Editorial Inde.

Vanden Auweele, I., Cuyper, B., Van Mele, V. y Rzewnicki, R. (1993). Elite performance and personality: from description and prediction to diagnosis and intervention. En R. N. Singer, M. Murphey y L. K. Tennant (Eds.), Handbook of Research on Sport Psychology (257-289). NY: McMillan Publishing Company

Vanyperen, N.W. (1994). Estrés psicosocial, apoyo parental, y rendimiento en jóvenes futbolistas con talento. Revista de Psicología del Deporte, 6, 119138.
Vealey, R.S. (2002). Personality and sport behavior. En T. S. Horn (ed.), Advances in sport psychology (2nd ed.) (pp.43-74). Champaign, IL: Human Kinetics.

Wann, D.L. (1997). Sport Psychology. Upper Saddle River: Prentice Hall.

Weinberg, R. y Gould, D. (2010). Fundamentos de Psicología del Deporte y del Ejercicio Físico. Madrid: Editorial Médica Panamericana.

Williams, C. A. y James, D. V. (2001). Science for Exercise and Sport. New York: Routledge.

(Artículo recibido: 06-02-2012; revisado: 18-02-2013; aceptado: 01-03-2013) 